\title{
Progressive Increases in Luminal Glucose Stimulate Proximal Sodium Absorption in Normal and Diabetic Rats
}

\author{
Norman Bank and Hagop S. Aynedjian \\ Renal Division, Department of Medicine, Montefiore Medical Center and the Albert Einstein College of Medicine, Bronx, New York 10467
}

\begin{abstract}
The effect of progressive increases in intraluminal glucose concentration on proximal tubule sodium absorption was studied in normal and streptozotocin diabetic rats by microperfusion. Each tubule was perfused twice, with and without glucose added to the perfusion fluid. Net sodium and water absorption were markedly enhanced by $300-500 \mathrm{mg} \%$ intraluminal glucose in both normal and diabetic rats. Substituting the transported but nonmetabolized glucose analogue, $\alpha$-methyl D-glucoside for glucose also resulted in marked stimulation of sodium absorption, whereas substituting bicarbonate and acetate for chloride in the perfusion solution inhibited the effect of glucose. These observations suggest that the stimulation of sodium absorption by glucose was mediated by the brush border $\mathrm{Na}$ /glucose cotransporter. Sodium concentration and osmolality were found to fall markedly to hypotonic levels when high glucose concentrations were in the perfusion fluid. This luminal hypotonicity may be an important driving force for proximal fluid absorption. In poorly controlled diabetes, high filtered glucose concentrations may lead to enhanced proximal sodium and water absorption, which could in turn contribute to volume expansion, hypertension, and renal hypertrophy. (J. Clin. Invest. 1990. 86:309-316.) Key words: sodium-glucose cotransport - microperfusion - proximal tubule function - luminal osmolality
\end{abstract}

\section{Introduction}

There is substantial although not unanimous evidence that glucose stimulates fluid and sodium absorption by the normal kidney. In isolated perfused rat kidneys, addition of glucose to the perfusion fluid results in an increase in both sodium and water reabsorption (1-5). In isolated perfused rabbit proximal tubules, glucose added to the luminal fluid, but not to the bath fluid, increases fluid absorption and the transepithelial electrical potential (6). However, in several free-flow micropuncture studies in normal rats (7-10) and dogs (11), glucose infusion was found to inhibit proximal sodium and water absorption (11), to increase absorption (7), or to have no specific effect (8, 10). The reasons for these disparate results are not certain, but variations in GFR, which alter the delivery of both glucose and sodium to the tubule, and changes in extracellular volume, are two possibilities. No previous study of the effect of glucose on

Address reprint requests to Dr. Bank, Department of Medicine, Montefiore Medical Center, 110 East 210th Street, Bronx, NY 10467.

Received for publication 15 September 1989 and in revised form 29 December 1989.

J. Clin. Invest.

(c) The American Society for Clinical Investigation, Inc. 0021-9738/90/07/0309/08 \$2.00

Volume 86, July 1990, 309-316 proximal tubule sodium reabsorption has been reported in diabetic animals.

The present experiments were undertaken to examine the effects of increasing concentrations of luminal D-glucose on proximal tubule sodium absorption in both normal and insulin-deficient diabetic rats. The in vivo reperfusion technique was used in which each tubule serves as its own control (12). This experimental approach obviates variations in GFR and allows precise control of delivery rate and perfused glucose concentrations. The findings indicate that in both normal and diabetic rats, progressive increases in luminal D-glucose stimulate net sodium and water absorption markedly. This was accompanied by a fall in sodium and glucose concentrations in the collected fluid, and a decrease in osmolality to frankly hypotonic levels. The enhancement of net sodium transport was presumably due to brush border $\mathrm{Na}$ /glucose cotransport since the nonmetabolized analogue $\alpha$-methyl D-glucoside also stimulated absorption. The findings are consistent with the hypothesis that in poorly controlled diabetes, high concentrations of filtered glucose may increase proximal sodium and water absorption, contributing to volume expansion, hypertension, and renal hypertrophy.

\section{Methods}

47 male Sprague-Dawley rats, weighing 275-325 g were studied. 15 rats were made diabetic by injection of streptozotocin $65 \mathrm{mg} / \mathrm{kg}$ body wt i.v. 10-14 d before study. These animals were provided with a regular rat pellet diet and unlimited amounts of drinking water, but they received no insulin. The remaining 32 rats were normal animals. On the day of micropuncture study, all animals were anesthetized with inactin, $100 \mathrm{mg} / \mathrm{kg}$ body wt, the trachea was intubated, and a jugular vein was cannulated with PE 50 tubing for constant infusion of Ringer's lactate solution. The left kidney was surgically exposed, cleaned of perirenal fat tissue, and immobilized in a lucite cup, as previously described (12-14).

Group 1. In 18 normal and 15 diabetic rats, microperfusion of surface proximal convoluted tubules was carried out under direct visualization via a dissecting stereomicroscope. The technique of reperfusion was used (12), in which each segment of tubule was perfused twice, once with an electrolyte solution devoid of glucose and once with the same solution containing 100,300 , or $500 \mathrm{mg} \%$ D-glucose. Both collected perfusates were measured for volume, $\left[{ }^{14} \mathrm{C}\right]$ inulin concentration and sodium concentration. The technique was as follows: an intravenous bolus injection of FD and $C$ green dye (Keystone Aniline and Chemical, Chicago, IL) was given, and an early proximal convolution was identified by direct visualization of appearance of the dye. A micropipette filled with stained castor oil, but with a small amount of F D and $C$ green-colored saline in the tip, was inserted into an early proximal convolution. The colored saline was injected into the lumen, and convolutions of the same tubule were identified by following the dye. A short column of castor oil was then injected into the lumen from the same pipette, after which this pipette was withdrawn and used to puncture several holes proximal to the oil block to allow escape of fluid coming from the glomerulus. Microinfusion of the tubule was begun by inserting a second pipette just distal to this oil block. The pipette 
contained the basic perfusion solution, the composition of which was as follows: sodium, $140 \mathrm{mM}$; bicarbonate $25 \mathrm{mM}$; chloride $118 \mathrm{mM}$; potassium $5 \mathrm{mM}$; calcium $1.5 \mathrm{mM}$; phosphate $2.5 \mathrm{mM}$. $\left[{ }^{14} \mathrm{C}\right]$ Inulin was added in trace amounts. Osmolality was $275 \mathrm{mosmol} / \mathrm{kg} \mathrm{H}_{2} \mathrm{O}$. The rate of microinfusion was controlled by a Sage pump which was calibrated to deliver $20 \mathrm{nl} / \mathrm{min}$. After 1-2 min of microinfusion, allowing the fluid to flow through the nephron, a second oil-filled (collecting) pipette was inserted into a segment of the tubule several convolutions distal to the proximal pipette. A second (distal) oil block was injected from this pipette. The flow of perfusion fluid was unavoidably interrupted for 5-10 s during injection of the distal oil block. A timed collection of the perfusion fluid was then made into the second pipette. After the collection was completed, the perfusion and collection pipettes were withdrawn. When several tubules had been perfused with the same solution, and their location on the surface of the kidney drawn, the perfusion pipette was filled with the same solution to which 100,300 , or $500 \mathrm{mg} \%$ D-glucose had been added. Each tubule was then reperfused with this solution, using the same technique. Proximal and distal oil blocks were reinjected if necessary. In each animal, three or four tubules were each perfused twice. In two normal and seven diabetic rats the sequence of microperfusion was reversed, i.e., the first solution contained glucose and the second was devoid of glucose. The following calculations were carried out:

perfusion rate $=(C / I)_{1 \mathrm{n}} \times$ collected volume $/ \mathrm{min}$

where $(\mathrm{C} / \mathrm{I})_{\ln }$ is the concentration ratio of $\left[{ }^{14} \mathrm{C}\right]$ inulin in the collected/ infused solutions.

absolute $\mathrm{H}_{2} \mathrm{O}$ absorbed $=$ perfusion rate $\times\left[1-(\mathrm{I} / \mathrm{C})_{\mathrm{ln}}\right]$.

Sodium concentration in the collected perfusion solution was measured in triplicate by a helium glow photometer. $\left[{ }^{14} \mathrm{C}\right]$ Inulin concentration was measured by liquid scintillation counting. Absolute net sodium absorption was calculated by the equation

$\mathrm{Na}^{+}$abs $=$Perfusion rate $\times\left[\mathrm{Na}_{i}^{+}\right]-$collection rate $\times\left[\mathrm{Na}_{\mathrm{c}}^{+}\right]$

where $\left[\mathrm{Na}_{i}\right]$ is the concentration in the initial perfusion fluid and $\left[\mathrm{Na}_{\mathrm{c}}^{+}\right]$ is the concentration in the collected fluid. Because each tubule served as its own control, the length of tubule for each paired perfusion was constant, and therefore microdissection of tubules to determine length was not carried out.

In the experiments in normal rats in which either $300 \mathrm{mg} \%$ or 500 $\mathrm{mg} \%$ D-glucose was used in the second of the paired tubular perfusions, blood glucose of the animals was raised by intravenous bolus infusion of 0.4 to $0.6 \mathrm{ml}$ of a hypertonic glucose solution $(500 \mathrm{mg} / \mathrm{ml})$ before starting the second perfusion of the tubules. This was followed by continuous intravenous infusion of either $300 \mathrm{mg} \%$ or $500 \mathrm{mg} \%$ glucose in Ringer's solution at $2.5 \mathrm{ml} / \mathrm{h}$. The purpose was to raise blood glucose to a level approximately the same as the intraluminal glucose concentration in order to minimize transepithelial osmotic gradients. However, in the two normal rats in which the order of microperfusion of the tubules was reversed, glucose was not administered intravenously. Also, in the diabetic animals, no attempt was made to modify the blood glucose concentration.

Group 2: quantitative glucose vs. sodium absorption. In three additional normal rats, sodium and glucose reabsorption were measured simultaneously in the same nephron segments with the reperfusion technique. In these experiments, the tubules were perfused first with the electrolyte solution containing $100 \mathrm{mg} \%$ D-glucose, and then they were reperfused with the same solution containing $500 \mathrm{mg} \%$ D-glucose. Intravenous glucose was not administered in these experiments, but rather blood glucose remained constant during both periods of the experiments. Trace amounts of $\left[{ }^{3} \mathrm{H}\right]$ methoxy inulin and $\left[{ }^{14} \mathrm{C}\right] \mathrm{D}$-glucose were added to the perfusion solutions. Sodium concentration in the collected perfusion solution was measured by the helium glow photometer method, and absolute rates of sodium absorption were calculated by Eq. 3. Absolute net glucose absorption was calculated by the equation:
Glucose absorption $(\mathrm{pmol} / \mathrm{min})=[$ Perfusion rate

$\times$ glucose concentration $] \times\left[1-\left(\mathrm{C} / \mathrm{I}_{14 \mathrm{C}} / \mathrm{C} / \mathrm{I}_{3 \mathrm{H}}\right)\right]$

where $C / I_{14 c}$ is the ratio of $\left[{ }^{14} \mathrm{C}\right]$ glucose in collected fluid/injected fluid, and $C / I_{3 \mathrm{H}}$ is the ratio of $\left[{ }^{3} \mathrm{H}\right]$ inulin in the collected/injected fluid. The glucose concentration in the perfusion fluid is expressed in $\mathrm{pmol} / \mathrm{nl}$ and the calculated glucose absorption is expressed as $\mathrm{pmol} / \mathrm{min}$. The molar concentration of radiolabeled glucose added to the perfusion solution was $<0.15 \mathrm{mM}$ in all experiments and this amount has been ignored in the calculations of unlabeled glucose absorption.

Group 3: effect of intraluminal anions. To study the effect of intraluminal anions on quantitative sodium and glucose absorption, paired microperfusions were carried out in four normal rats with a solution devoid of chloride and of the following composition: sodium $140 \mathrm{mM}$, bicarbonate $74 \mathrm{mM}$, acetate $70 \mathrm{mM}$, potassium $4 \mathrm{mM}$, calcium phosphate $2.5 \mathrm{mM}$, osmolality $275 \mathrm{mosmol}$. Since both bicarbonate and acetate are largely converted to non-ionic forms before traversing the brush border membrane, this perfusion fluid provided little or no permeant anions to accompany the absorbed sodium (15). The first of the paired perfusion solution contained $100 \mathrm{mg} \%$ D-glucose and the second solution contained $500 \mathrm{mg} \%$ D-glucose. As in group 2, glucose was not administered intravenously during the second period of the experiments, allowing blood glucose to remain constant. $\left[{ }^{14} \mathrm{C}\right]$ Glucose and $\left[{ }^{3} \mathrm{H}\right]$ inulin were added to the perfusion solutions in trace amounts, as described above. The technique of microperfusion was the same as in the preceding groups.

Group 4: $\alpha$ methyl D-glucoside. In three normal rats, reperfusion of proximal tubules was carried out with the same electrolyte solution used above to which $27.5 \mathrm{mM} \alpha$-methyl D-glucoside (Sigma Chemical Co., St. Louis, MO) was added, instead of D-glucose. In these experiments the first perfusion solution contained no sugar, and the second contained the $\alpha$-methyl D-glucoside. Water absorption was measured with $\left[{ }^{14} \mathrm{C}\right]$ inulin and sodium concentration in the collected fluid was measured by helium glow photometry, as described above. No glucose was administered intravenously.

Group 5: osmolality measurements. In four animals, osmolality of plasma, perfusion solutions, and collected perfusates was measured by the freezing point method of Ramsay and Brown, as described previously $(16,17)$. The accuracy of this method is \pm 2 mosmol $(16)$. Two different perfusion solutions were used: $(a)$ the basic electrolyte solution $+500 \mathrm{mg} \%$ D-glucose; $(b) \mathrm{NaCl} 155 \mathrm{mM}$. The rate of perfusion was $20 \mathrm{nl} / \mathrm{min}$, as in the preceding experiments. A total of 21 proximal tubules were studied.

In all experiments, blood glucose was measured enzymatically with a glucose meter (Beckman Instrument Co., Inc., Fullerton, CA). Statistical analysis was by paired Student's $t$ test for the reperfusion samples, and by unpaired $t$ test for other comparisons.

\section{Results}

In Table I are shown the data for blood glucose concentrations in the normal and diabetic rats, comparing these values with the concentration of glucose in the various perfusion solutions. In the normal rats when either 300 or $500 \mathrm{mg} \%$ glucose was in

Table I. Blood Glucose and Perfusion Fluid Glucose

\begin{tabular}{llccccc}
\hline \multicolumn{1}{c}{ Group } & Blood & Perfusion & Blood & Perfusion & Blood & Perfusion \\
\hline & & \multicolumn{7}{c}{$m g \%$} \\
Normal rats & $117 \pm 5$ & 100 & $297 \pm 16^{*}$ & 300 & $420 \pm 14^{*}$ & 500 \\
Diabetic rats & $384 \pm 31$ & 100 & $369 \pm 16$ & 300 & $438 \pm 6$ & 500 \\
\hline
\end{tabular}

* In normal rats, blood glucose raised by intravenous glucose infusion. 
Table II. Effect of Intraluminal Glucose on Proximal Water Absorption (nl/min)

\begin{tabular}{|c|c|c|c|c|c|c|}
\hline \multirow[b]{2}{*}{ Group } & \multicolumn{2}{|c|}{ Glucose (mg\%) } & \multicolumn{2}{|c|}{ Glucose (mg\%) } & \multicolumn{2}{|c|}{ Glucose (mg\%) } \\
\hline & 0 & 100 & 0 & 300 & 0 & 500 \\
\hline & \multicolumn{6}{|c|}{$\mathrm{nl} / \mathrm{min}$} \\
\hline \multirow[t]{2}{*}{ Normal rats } & $1.30 \pm 0.16$ & $1.88 \pm 0.18^{*}$ & $1.58 \pm 0.16$ & $2.29 \pm 0.22^{*}$ & $1.81 \pm 0.16$ & $2.56 \pm 0.15^{*}$ \\
\hline & \multicolumn{2}{|c|}{$n=15$} & \multicolumn{2}{|c|}{$n=17$} & $n=20$ & \\
\hline \multirow[t]{2}{*}{ Diabetic rats } & $1.80 \pm 0.16$ & $2.61 \pm 0.11^{*}$ & $1.65 \pm 0.17$ & $2.67 \pm 0.16^{*}$ & $2.02 \pm 0.11$ & $2.86 \pm 0.14^{*}$ \\
\hline & \multicolumn{2}{|c|}{$n=17$} & \multicolumn{2}{|c|}{$n=13$} & \multicolumn{2}{|c|}{$n=15$} \\
\hline
\end{tabular}

Data expressed as mean \pm SE. Each tubule was perfused twice. ${ }^{*} P<0.001$ by paired $t$ test, experimental vs. control. $n$, number of tubules perfused. $N$, number of animals.

the second of the paired perfusion solutions, i.e., the second half of the experiment, blood glucose was raised by intravenous infusion of hypertonic glucose. In the diabetic rats, no attempt was made to alter blood glucose. As can be seen, the transepithelial gradients of glucose varied considerably among the various experiments. In the normal rats, there were relatively small glucose gradients during perfusion with glucosecontaining fluid, and thus transepithelial osmotic gradients due to glucose were minimized. However, each tubule was also perfused with a glucose-free solution, which was lower in osmolality, and under these circumstances plasma osmolality was higher than luminal osmolality. In the diabetic rats, blood glucose was considerably higher than luminal glucose in two of the three experimental conditions. Moreover, when these tubules were perfused with fluid devoid of glucose, an even larger osmotic gradient existed across the tubular epithelium. Thus, although an attempt was made to minimize transepithelial glucose gradients during perfusion with glucose containing solutions, a large osmotic gradient usually existed during the first perfusion when no glucose was in the lumen. This gradient was diminished during the second perfusion by addition of glucose to the perfusion solution.

Group 1: water and sodium absorption (Tables II and III, Figs. 1-3). The data for proximal water absorption are presented in Table II, in which the paired microperfusions are grouped according to the concentration of glucose added to the perfusion solution. The data have been analyzed by paired $t$ test for individual tubules. As can be seen, in both the normal and the diabetic animals, water absorption increased significantly when glucose was added to the perfusion fluid. This was observed in every tubule studied. Since the transepithelial os- motic gradient favoring fluid absorption was less during the glucose perfusion than during the control perfusion, the enhanced fluid absorption cannot be attributed to a more favorable osmotic gradient. The increments in fluid absorption are shown in Fig. 1. Although these increments appear to be greater in the diabetic rats, a statistical comparison with the normals is not justified because tubular lengths were not determined, and renal hypertrophy with increased tubular length and diameter had most likely occurred (13).

The concentrations of sodium in the collected perfusates are shown in Fig. 2. In the normal rats, addition of increasing amounts of glucose to the perfusion fluid resulted in a progressive fall in intraluminal sodium concentration to a nadir of $112 \mathrm{meq} / \mathrm{liter}$ reached with the $300 \mathrm{mg} \%$ solution. No further fall in $\left[\mathrm{Na}^{+}\right]$was observed with the $500 \mathrm{mg} \%$ glucose solution. In the diabetic rats, intraluminal $\left[\mathrm{Na}^{+}\right]$also fell with increasing perfusion fluid glucose concentrations. The average sodium concentrations tended to be slightly higher in the diabetic rats than in the normals, even though tubular diameters and lengths were most likely greater. A larger luminal surface area for sodium transport would be expected in the diabetic animals. The fact that the average sodium concentrations in the collected perfusates tended to be higher than in the normals is consistent with some impairment in establishing a transepithelial sodium gradient in the diabetic animals.

The measured rates of sodium absorption are shown in Table III. In both the normal and diabetic rats, proximal sodium absorption increased strikingly when each of the three D-glucose concentrations was added to the perfusion solution. This occurred in every nephron studied in both the normal and diabetic rats. The increment in sodium absorption with

Table III. Effect of Intraluminal Glucose on Proximal Sodium Absorption

\begin{tabular}{|c|c|c|c|c|c|c|}
\hline \multirow[b]{2}{*}{ Group } & \multicolumn{2}{|c|}{ Glucose } & \multicolumn{2}{|c|}{ Glucose } & \multicolumn{2}{|c|}{ Glucose } \\
\hline & 0 & 100 & 0 & 300 & 0 & 500 \\
\hline & \multicolumn{6}{|c|}{$m g \%$} \\
\hline Normal rats & $265 \pm 22$ & $447 \pm 32^{*}$ & $312 \pm 16$ & $833 \pm 26^{*}$ & $326 \pm 26$ & $861 \pm 17^{*}$ \\
\hline Diabetic rats & $247 \pm 25$ & $424 \pm 13^{*}$ & $268 \pm 24$ & $719 \pm 26^{*}$ & $306 \pm 14$ & $880 \pm 24^{*}$ \\
\hline
\end{tabular}

Data expressed as pmol/min, mean \pm SE. ${ }^{*} P<0.001$ experimental vs. control, paired $t$ test. $N$ and $n$ same as in Table II. 


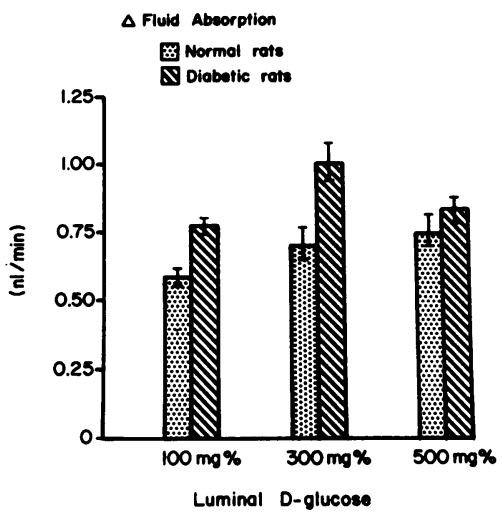

Figure 1. Increments in fluid absorption when D-glucose was added to a glucose-free electrolyte perfusion solution.

addition of glucose is shown in Fig. 3. It appears that a maximum stimulation of sodium absorption was achieved with 300 $\mathrm{mg} \%$ D-glucose in the normal rats, but in the diabetics 500 mg\% D-glucose seemed to cause a slightly greater stimulus than $\mathbf{3 0 0} \mathrm{mg} \%$. However, as noted above, sodium transport in the diabetic rats might be impaired if expressed in terms of luminal surface area, as hypertrophy of the tubules was most likely present. It is important to note that the increment in sodium absorption occurred in spite of the higher luminal osmolality due to added glucose, and thus the stimulation of sodium transport cannot be attributed to osmotically induced fluid transfer. In the diabetic rats, blood glucose was in a hyperglycemic range and remained relatively constant throughout each experiment. Our observations on enhanced sodium absorption in normal rats are in accord with the derived values for sodium absorption during hyperglycemia calculated by Von Baeyer et al. (18).

Group 2: simultaneous quantitative sodium and glucose absorption (Figs. 4 and 5). The reperfusion technique was used to compare the effect of increasing glucose concentration on absolute rates of net glucose and sodium absorption simultaneously in the same proximal convoluted tubules. In these experiments, the first perfusion solution contained $100 \mathrm{mg} \%$ D-glucose and the second perfusion fluid contained $500 \mathrm{mg} \%$ D-glucose. $\left[{ }^{3} \mathrm{H}\right]$ methoxy inulin and $\left[{ }^{14} \mathrm{C}\right]$ glucose were added in trace amounts. It is clear from the results shown in Fig. 4 that both glucose and sodium absorption increased in every tubule

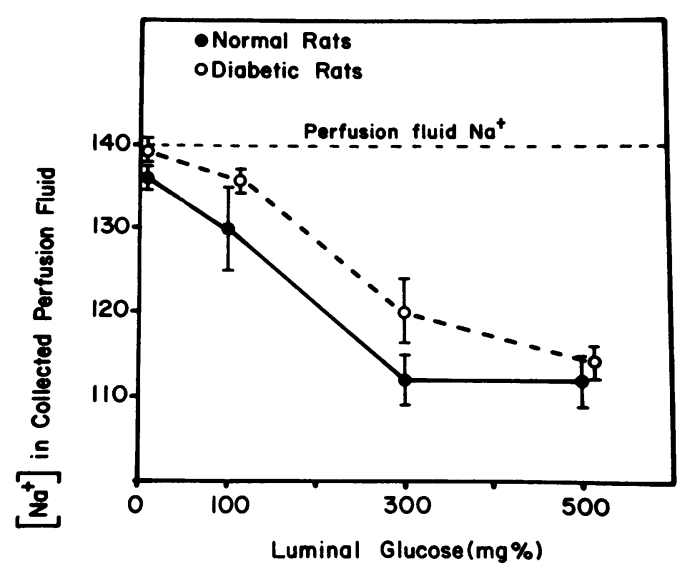

Figure 2. Concentration of sodium in collected perfusates (milliequivalents per liter). Data shown as mean \pm SEM.

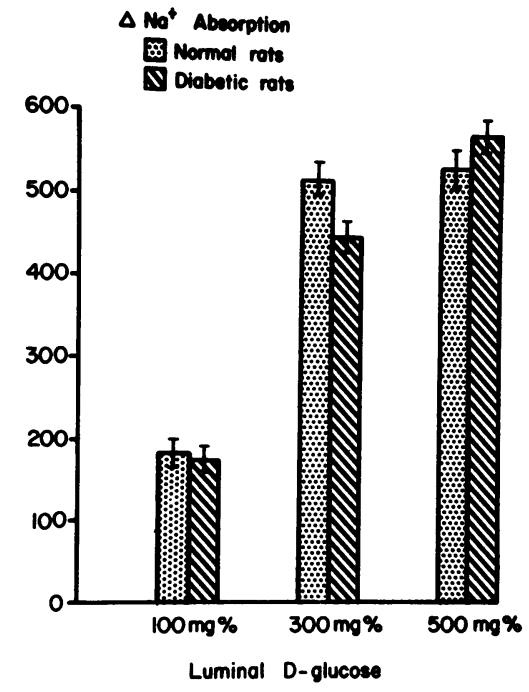

Figure 3. Increments in net sodium absorption when D-glucose was added to a glucose-free electrolyte perfusion solution (picomoles per minute). when the perfusion fluid glucose concentration was raised from 100 to $500 \mathrm{mg} \%$. Previous studies have shown that net glucose absorption by the proximal tubule can be accounted for by an active efflux mechanism and a small passive backflux $(19,20)$, the passive backflux being dependent upon the blood/lumen concentration gradient. Since in our experiments, the transepithelial glucose gradient usually favored efflux (Table I), especially when large amounts of glucose were added to the lumen, the reabsorption of glucose most likely reflects principally active efflux with little or no passive influx. As shown in Fig. 4, the increment in net sodium absorption appeared to be much greater than the increment in glucose absorption. In order to examine this relationship more closely, the increment in absorption in each tubule, i.e., $\Delta$ glucose vs. $\Delta$ sodium, has been plotted in Fig. 5 . Each closed circle represents a single tubule, microperfused twice. The solid line is the line of identity, i.e., points would fall along this line if sodium and glucose absorption increased by equal amounts. It is evi-

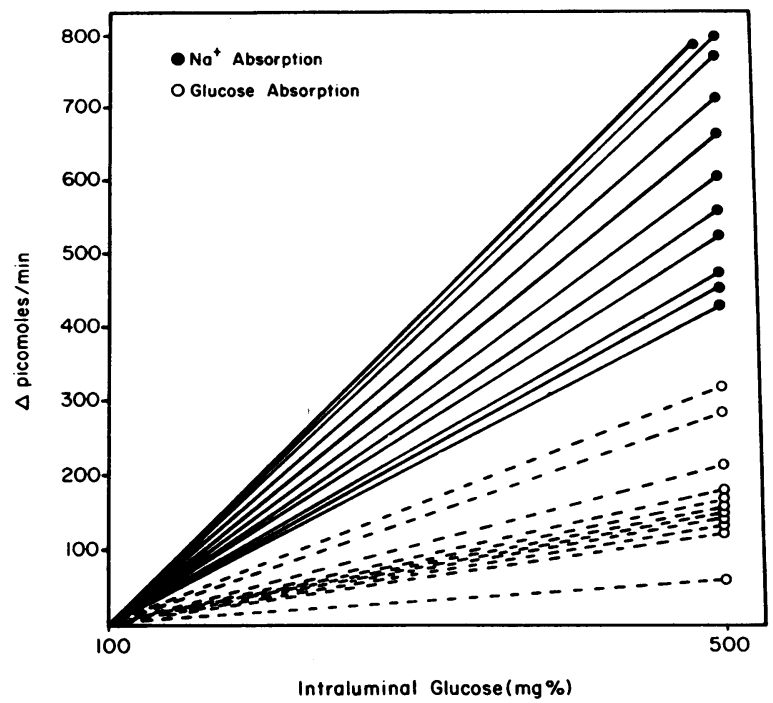

Figure 4. Paired absolute rates of sodium and glucose absorption with 100 or $500 \mathrm{mg} \%$ glucose in perfusion solution. Sodium and glucose absorption measured in same tubules. 


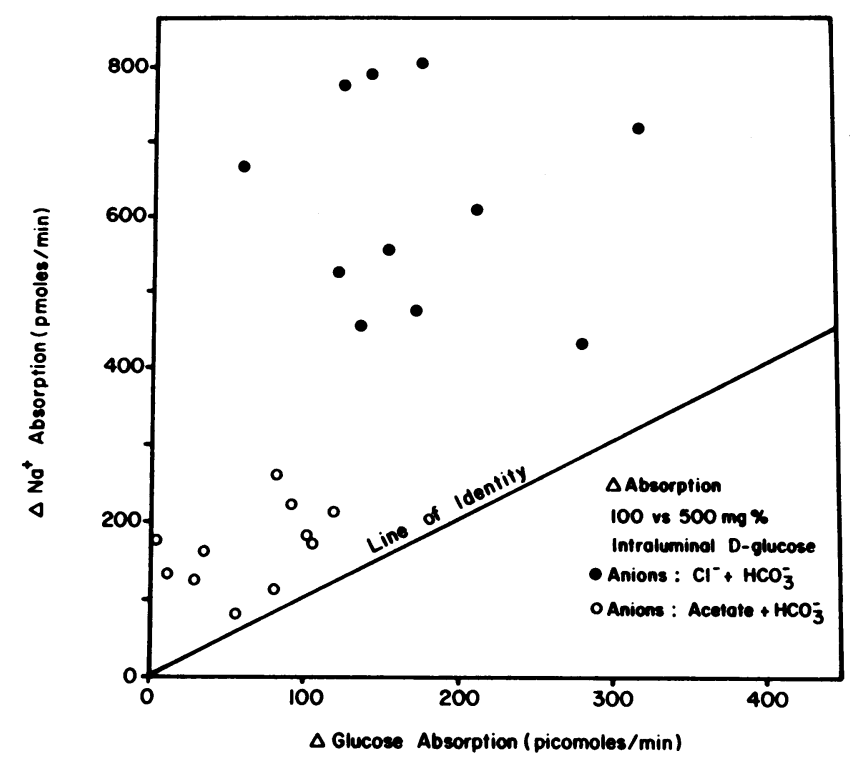

Figure 5. Increment in sodium and glucose absorption with 500 vs. $100 \mathrm{mg} \%$ glucose in perfusion solution. Each point represents a single tubule perfused twice. Closed and open circles reflect anion composition of perfusion solution.

dent that in individual tubules, sodium absorption increased considerably more than glucose absorption. The relationship varied from $\sim 2: 1$ to $8: 1$ in different tubules. There was no statistically significant linear correlation of the data $(r=0.1)$.

Group 3: effect of intraluminal anions (Table IV, Fig. 5). Studies of $\mathrm{Na}$ /glucose cotransport in isolated brush border membrane vesicles have shown that a permeant anion is necessary in the bathing medium in order for the transporter to operate $(15,21)$. Bicarbonate and acetate in the bathing media severely reduce the rapid entry of sodium into the vesicles when glucose is added $(15,21)$. In the group 3 animals, we examined the effect of substituting bicarbonate and acetate for chloride in the luminal perfusion fluid. As shown in Table IV, net absorption of both sodium and water were much lower in these experiments than in group 1 in which chloride was the major anion in the perfusion fluid (Tables II and III). The concentration of sodium in the collected perfusate fell from $130 \pm 1.1$ with $100 \mathrm{mg} \%$ glucose to $118 \pm 1.2 \mathrm{meq} / \mathrm{liter}$ when $500 \mathrm{mg} \%$ D-glucose was in the perfusion solution $(P<0.001)$.

Table IV. Sodium and Glucose Absorption with Acetate + Bicarbonate Anion Solution

\begin{tabular}{ccccc}
\hline $\begin{array}{c}\text { Perfusion } \\
\text { solution glucose }\end{array}$ & $\begin{array}{c}\left.\mathrm{Na}^{+}\right] \text {Collected } \\
\text { perfusate }\end{array}$ & $\begin{array}{c}\text { Water } \\
\text { absorption }\end{array}$ & $\begin{array}{c}\mathrm{Na}^{+} \\
\text {absorption }\end{array}$ & $\begin{array}{c}\text { Glucose } \\
\text { absorption }\end{array}$ \\
\hline$m M$ & $m M$ & $n l / m i n$ & \multicolumn{2}{c}{$p m o l / m i n$} \\
5.55 & $129.8 \pm 1.1$ & $0.403 \pm 0.316$ & $263 \pm 33$ & $26.4 \pm 2.3$ \\
$\left(\begin{array}{c}100 \mathrm{mg} \%) \\
27.8\end{array}\right.$ & $118.4 \pm 1.2$ & $0.135 \pm 0.284$ & $422 \pm 33$ & $93.8 \pm 12.3$ \\
$(500 \mathrm{mg})$ & & NS & $<0.001$ & $<0.001$ \\
$P$ & $<0.001$ & & &
\end{tabular}

Statistical analysis by Student's paired $t$ test.

$N, 4$.

$n, 11$.
It should be noted that fluid absorption was not stimulated by the higher glucose concentration, and in fact the mean value was lower $(P=\mathrm{NS})$. The quantitative increments of sodium and glucose absorption in individual tubules is shown in Fig. 5 as open circles. As can be seen, very little stimulation of sodium or glucose absorption occurred when glucose was raised from 100 to $500 \mathrm{mg} \%$, in contrast to the experiments in which chloride was the major intraluminal anion. Thus, the $\mathrm{Na} / \mathrm{glu}-$ cose cotransporter in the intact proximal tubule appeared to behave similarly to that in isolated brush border membrane vesicles with regard to requiring a permeant charge-carrying anion.

Group 4: effect of $\alpha$-methyl D-glucoside. In additional normal rats, reperfusion of proximal tubules was carried out with 0 or $27.5 \mathrm{mM} \alpha$-methyl D-glucoside added to the basic perfusion solution. Sodium concentration in the collected perfusion fluid fell to $110 \mathrm{meq} / \mathrm{liter}$ when $27.5 \mathrm{M} \alpha$-methyl D-glucoside was in the perfusion fluid, similar to the effect of D-glucose. Sodium absorption rose markedly from $320 \pm 15 \mathrm{pmol} / \mathrm{min}$ (zero sugar) to $725 \pm 30 \mathrm{pmol} / \mathrm{min}$, yielding an increment of $405 \mathrm{pmol} / \mathrm{min}$. This appears to be a somewhat smaller increment than that observed with D-glucose in the normal and diabetic rats (Fig. 3), but a statistical comparison between the groups cannot be made because tubular lengths and surface areas were not evaluated. Nevertheless, the observations with $\alpha$-methyl D-glucoside support the role of the $\mathrm{Na} /$ glucose cotransporter in the enhanced sodium absorption. A smaller stimulus with this glucose analogue is consistent with its lower affinity for the transporter $(22,23)$.

Group 5: osmolality measurements. In Fig. 6 are shown the osmolality data for initial perfusion fluid, and collected perfusate when two different perfusion solutions were used. One solution was the basic electrolyte perfusion solution with 500 $\mathrm{mg} \%$ D-glucose added. The other was a solution of $155 \mathrm{mM}$ $\mathrm{NaCl}$, devoid of glucose. Plasma osmolality in these experiments is also shown. The initial osmolality of the glucose-containing solution was 299 mosmol, close to that of plasma. The osmolality of the $\mathrm{NaCl}$ solution was 288 mosmol, significantly lower than plasma. The osmolality of the glucose-containing solution fell to $277.5 \pm 1.5$ mosmol $(\Delta 22$ mosmol) when perfused into the proximal tubule. In contrast, the osmolality of

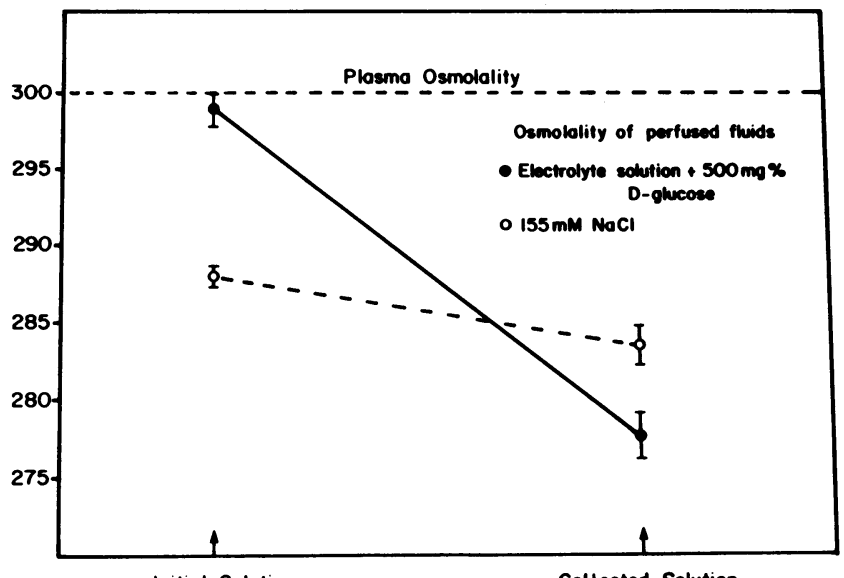

Figure 6. Initial osmolality and final osmolality of glucose-containing and glucose-free perfusion solutions. Data are expressed as milliosmoles per liter. 
the $\mathrm{NaCl}$ solution fell by only 4.7 mosmol, to $283.3 \pm 1.3$ mosmol. The difference in final osmolality of the two collected fluids was statistically significant $(P<0.02)$, as was the fall in osmolality.

\section{Discussion}

The present microperfusion experiments demonstrate that in both normal and diabetic rats, stepwise increases in luminal glucose concentration markedly stimulate sodium and water absorption. This was accompanied by a striking fall in intraluminal sodium concentration, and a 22 mosmol fall in osmolality to a level frankly hypotonic to plasma. In contrast, perfusion with a $\mathrm{NaCl}$ solution devoid of glucose resulted in a small but significant fall in osmolality of 4 mosmol.

Several mechanisms have been proposed to explain the effect of glucose to enhance renal sodium absorption (24): stimulation of $\mathrm{Na}$ /glucose cotransport, solvent drag, and metabolic energy provided by absorbed glucose. We carried out experiments to examine these various mechanisms. Solvent drag did not seem to play a significant role, since sodium absorption increased whether transepithelial glucose gradients favored influx or efflux of glucose. In fact, in the group 1 experiments, no glucose was present in the first perfusion, and glucose was added to the second of the paired perfusions. Thus, whatever the plasma glucose concentration, the gradient across the tubular epithelium was diminished during the second perfusion. In spite of this, both sodium and water absorption were increased by the addition of intraluminal glucose. In addition, in groups 2-5, glucose was not administered intravenously but rather plasma glucose remained constant throughout both periods of the experiment. In the diabetic rats, plasma glucose was also constant throughout the experiment, yet sodium and water absorption were markedly increased when glucose was added to the luminal perfusion solution. It seems highly unlikely that passive diffusion of glucose from lumen to blood can account for the observed increase in fluid and sodium absorption, since the rat proximal tubule has a low permeability to glucose (25). Our observations strongly suggest therefore that the effect of glucose in these experiments was mediated from the luminal side of the tubule by stimulating transport. In agreement with this conclusion, Burg et al. (6) found in isolated perfused rabbit proximal tubules that addition of glucose to the bathing media had no effect on fluid absorption, whereas addition to the luminal fluid stimulated fluid absorption. Finally, the fact that osmolality of the luminal fluid fell with addition of glucose to the perfusion fluid suggests that the primary event was active transport of solute out of the lumen.

We examined the role of the $\mathrm{Na}$ /glucose cotransporter in two protocols. Substitution of $\alpha$-methyl D-glucoside, a glucose analogue which substitutes for glucose on the sodium/glucose co-transporter but is not metabolized $(22,24)$, stimulated sodium absorption markedly, almost to the same degree as Dglucose. These observations are in agreement with those of Burg et al. (6), and provide evidence for $\mathrm{Na}$ /glucose cotransport and against a metabolic effect of glucose.

A second protocol examined the effect of anions on glucose stimulated sodium and water absorption. A characteristic in vitro property of the $\mathrm{Na}$ /glucose cotransporter is that rapid uptake of sodium or glucose from the medium (overshoot) requires the presence of an anion in the bathing solution which readily permeates the transporting membrane $(15,21)$. Nonpermeating anions such as sulfate or ferrocyanide prevent the rapid uptake of either sodium or glucose. Similarly, anions that are converted into non-ionic forms before permeating membranes, such as bicarbonate and acetate, markedly impede $\mathrm{Na}$ /glucose cotransport, presumably via a membrane potential effect (15). In the group 4 experiments (Table IV, Fig. 5 ), chloride was replaced by bicarbonate and acetate in the perfusion fluid. It is clear that both sodium and glucose absorption were markedly reduced under these experimental conditions. Raising glucose to $500 \mathrm{mg} \%$ in the perfusion fluid had only a small effect on sodium and glucose absorption (Fig. 5). Measurements of membrane potentials in proximal tubules have shown that when glucose is added to the lumen, depolarization of the luminal membrane occurs, most likely due to sodium entry via $\mathrm{Na}$ /glucose cotransport $(26,27)$. Ordinarily, this depolarization would be minimized by entry of an anion from the lumen which would tend to restore intracellular electronegativity. In the absence of a permeant anion, sodium entry via $\mathrm{Na} / g l u c o s e$ cotransport would be expected to cause more marked depolarization of the brush border membrane. This in turn could severely reduce the electrochemical driving force for further passive sodium entry across the brush border membrane. Thus, the observations made in the group 4 animals are in accord with the in vitro data from isolated brush border membrane vesicles, and add further support to the view that $\mathrm{Na}$ /glucose cotransport was largely responsible for our observations.

From the above considerations, we conclude that the main mechanism responsible for the stimulation of sodium absorption by glucose was the $\mathrm{Na}$ /glucose cotransporter. However, in apparent discord with this conclusion is the fact that in isolated brush border membrane preparations of cortical tubules, the cotransporter operates in a 1:1 stoichiometry (28). In the outer medulla of the kidney, the co-transporter has been found to have a stoichiometry of 2:1 (29). As shown in Figs. 4 and 5, addition of glucose to the lumen stimulated sodium absorption considerably more than glucose absorption, and furthermore no consistent ratio between these two was observed. In these normal rats, insulin may have acted to stimulate basolateral sodium transport $(30,31)$, independent of the effect of intraluminal glucose on the brush border $\mathrm{Na}$ /glucose cotransporter. However, since glucose was not administered intravenously in these experiments, it seems unlikely that endogenous insulin was stimulated to higher than fasting levels. A discrepancy appears to exist between the intact nephron and isolated brush border membrane experiments with regard to the stoichiometry. The reason for this disagreement is not clear.

Recently, several investigators have reported that signifcant degrees of luminal hypotonicity can be generated and sustained by the rat proximal tubule (32-34). Hypotonicity of approximately -2 to -20 mosmol was measured under freeflow and microperfusion experimental conditions. Liu et al. (33) found an average decrease in osmolality along the length of the proximal tubule of $1 \mathrm{mosmol} / \mathrm{mm}$ length under freeflow conditions. It has been postulated that proximal fluid absorption depends upon the development of intraluminal hypotonicity, relative to plasma (32-34), in that the critical osmotic force for fluid absorption is due to luminal hypotonicity, rather than hypertonicity developing in the basolateral interspaces (32). In the present study, we found that addition of high concentrations of glucose to the perfusion fluid, resulted 
in the development of striking hypotonicity of the luminal fluid, due to enhanced sodium (anion) absorption. Presumably, this hypotonicity provided the driving force which increased absolute fluid absorption in both the normal and diabetic rats (Table II). An important role for glucose (and amino acids) in generating luminal hypotonicity was predicted by Green and Giebisch (32), although the degree of hypotonicity that developed with hyperglycemic levels of glucose is remarkable.

It should be noted that in the group 3 experiments in which bicarbonate and acetate were substituted for chloride in the perfusion solution, luminal $\left[\mathrm{Na}^{+}\right]$fell markedly, and presumably the fluid became hypotonic. However, this was not accompanied by enhanced fluid absorption (Table IV vs. Table II). In these experiments, sodium absorption was also much less than it was when chloride was in the perfusion solution. Thus, the effectiveness of intraluminal hypotonicity as a driving force for fluid absorption appears to be influenced by the anion composition of the luminal fluid and the rate of sodium absorption. As discussed above, $\mathrm{Na}$ /glucose cotransport is markedly constrained in the absence of a permeant anion (15), and this may have prevented fluid absorption in spite of luminal hypotonicity. Alternatively, high reflection coefficients for bicarbonate and acetate may have limited both sodium and water absorption.

Our observations suggest that high proximal tubular glucose concentrations in poorly controlled diabetes may lead to excessive reabsorption of sodium and water. This, in turn, could contribute to ECF volume expansion, hypertension, and renal hypertrophy $(35,36)$. It is clear, however, that enhanced proximal sodium and fluid absorption would have to be offset by decreased reabsorption at a more distal site to allow a steady state to be achieved. Atrial natriuretic peptide, which is elevated in diabetes $(37,38)$, would be a likely candidate for mediating decreased absorption distally.

In conclusion, our studies demonstrate that stepwise increases in intraluminal glucose concentration markedly stimulate net sodium absorption in both normal and diabetic proximal tubules. Luminal hypotonicity is generated by the presence of glucose, due to enhanced sodium absorption, and this presumably provides an important transepithelial osmotic driving force for reabsorption of fluid by the proximal tubule. In poorly controlled diabetes, high concentrations of glucose in the glomerular filtrate may lead to increased renal sodium and water absorption which in turn could contribute to volume expansion, hypertension, and renal hypertrophy.

\section{Acknowledgments}

This work was supported by a grant from the U. S. Public Health Service (DK 32469).

\section{References}

1. Trimble, M. E., and R. H. Bowman. 1973. Renal $\mathrm{Na}^{+}$and $\mathrm{K}^{+}$ transport: effects of glucose, palmitate, and $\alpha$-bromopalmitate. Am. J. Physiol. 225:1057-1062.

2. Trimble, M. E. 1976. Effects of L-glucose on sodium reabsorption in the isolated perfused rat kidney. Life Sci. 17:1799-1806.

3. Frega, N. S., J. M. Weinberg, B. D. Ross, and A. Leaf. 1977. Stimulation of sodium transport by glucose in the perfused rat kidney. Am. J. Physiol. 233(3):F235-F240.
4. Gregg, C. M., J. J. Cohen, A. J. Black, M. A. Espeland, and M. L. Feldstein. 1978. Effects of glucose and insulin on metabolism and function of perfused rat kidney. Am. J. Physiol. (Renal Fluid Electrolyte Physiol.) 4(1):F52-F61.

5. Cambier, P., and J. P. Godon. 1979/80. Dissociation of sodium and glucose transport in the isolated perfused rat kidney: Effect of lactate as a substrate. Renal Physiol. (Basel). 2:12-20.

6. Burg, M., C. Patlak, N. Green, and D. Villey. 1976. Organic solutes in fluid absorption by renal proximal convoluted tubules. $\mathrm{Am}$. J. Physiol. 231(2):627-637.

7. Weinman, E. J., W. N. Suki, and G. Eknoyan. 1976. D-glucose enhancement of water reabsorption in proximal tubule of the rat kidney. Am. J. Physiol. 231(3):777-780.

8. Bishop, J. H. V., R. Green, and S. Thomas. 1978. Effects of glucose on water and sodium reabsorption in the proximal convoluted tubule of rat kidney. J. Physiol. 275:481-493.

9. Knight, T. F., H. O. Senekjian, S. C. Sansom, and E. J. Weinman. 1980. Proximal tubule glucose efflux in the rat as a function of delivered load. Am. J. Physiol. 238(Renal Fluid Electrolyte Physiol. 7):F499-F503.

10. Bishop, J. H. V., R. Green, and S. Thomas. 1979. Free-flow reabsorption of glucose, sodium, osmoles and water in rat proximal convoluted tubule. J. Physiol. 288:331-351.

11. DeFronzo, R. A., M. Goldberg, and Z. S. Agus. 1976. The effects of glucose and insulin on renal electrolyte transport. J. Clin. Invest. 58:83-90.

12. Bank, N., H. S. Aynedjian, and B. F. Mutz. 1985. Evidence for a DCCD-sensitive component of proximal bicarbonate reabsorption. Am. J. Physiol. 249:F636-F644.

13. Bank, N., P. Mower, H. S. Aynedjian, B. M. Wilkes, and S. Silverman. 1989. Sorbinil prevents glomerular hyperperfusion in diabetic rats. Am. J. Physiol. 25(Renal Fluid Electrolyte Physiol. 25):F1000-F1006.

14. Bank, N., M. A. G. Lahorra, H. S. Aynedjian, and B. M. Wilkes. 1988. Sodium restriction corrects hyperfiltration of diabetes. Am. J. Physiol. 254:F668-F676.

15. Beck, J. C., and B. Sacktor. 1975. Energetics of the $\mathrm{Na}^{+}$-dependent transport of D-glucose in renal brush border membrane vesicles. J. Biol. Chem. 250:8674-8680.

16. Ramsay, J. A., and R. H. J. Brown. 1955. Simplified apparatus and procedure for freezing-point determinations upon small volumes of fluid. J. Sci. Instr. 32:372-375.

17. Bank, N., and H. S. Aynedjian. 1964. A micropuncture study of the renal concentrating defect of potassium depletion. Am. J. Physiol. 206:1347-1354.

18. Von Baeyer, H., D. A. Haeberle, J. B. Van Liew, and D. Hare. 1980. Glomerular tubular balance of renal D-glucose transport during hyperglycemia. Pfluegers Arch. Eur. J. Physiol. 384:39-47.

19. Von Baeyer, H., C. von Conta, D. Haeberle, and P. Deetjen. 1973. Determination of transport constants for glucose in proxima tubules of the rat kidney. Pfluegers Arch. Eur. J. Physiol. 343:273-286.

20. Barfuss, D. W., and J. A. Schafer. 1981. Differences in active and passive glucose transport along the proximal nephron. Am. $J$ Physiol. 240:F322-F332.

21. Hilden, S. A., and B. Sacktor. 1979. D-glucose-dependent sodium transport in renal brush border membrane vesicles. J. Biol. Chem. 254:7090-7096.

22. Aronson, P. S., and B. Sacktor. 1975 . The $\mathrm{Na}^{+}$gradient-dependent transport of D-glucose in renal brush border membranes. J. Biol. Chem. 250:6032-6039.

23. Ullrich, K. J., G. Rumrich, and S. Kloss. 1974. Specificity and sodium dependence of the active sugar transport in the proximal convolution of the rat kidney. Pfluegers Arch. Eur. J. Physiol. 351:35-48.

24. Ullrich, K. J. 1979. Sugar, amino acid, and $\mathrm{Na}^{+}$cotransport in the proximal tubule. Annu. Rev. Physiol. 41:181-195.

25. Warnock, D. G., C. S. Patlak, and M. B. Burg. 1978. Contribution of leaked load to solute transport by renal tubules. Am. J. Physiol. 234:F480-F484. 
26. Maruyama, T., and T. Hoshi. 1972. The effect of D-glucose on the electrical potential profile across the proximal tubule of newt kidney. Biochim. Biophys. Acta. 282:214-225.

27. Fromter, E., and K. Luer. 1973. Electrical studies on sugar transport kinetics of rat proximal tubule. Pfluegers Arch. Eur. J. Physiol. 343:R47.

28. Turner, R. J., and A. Moran. 1982. Stoichiometric studies of the renal outer cortical brush border membrane D-glucose transporter. J. Membr. Biol. 67:73-80.

29. Turner, R. J., and A. Moran. 1982. Further studies of proximal tubular brush border membrane D-glucose transport heterogeneity. $J$. Membr. Biol. 70:37-45.

30. Rostand, S. G., J. B. Watkins, and R. S. Clements, Jr. 1980. The effect of insulin and of anti-insulin serum on handling of sodium by the isolated, perfused kidney of the streptozotocin-diabetic rat. Diabetes. 29:679-685.

31. Baum, M. 1987. Insulin stimulates volume absorption in the rabbit proximal convoluted tubule. J. Clin. Invest. 79:1104-1109.

32. Green, R., and G. Giebisch. 1984. Luminal hypotonicity: a driving force for fluid absorption from the proximal tubule. Am. $J$. Physiol. 246(Renal Fluid Electrolyte Physiol. 15):F167-F174.

33. Liu, F-Y., M. G. Cogan, and F. C. Rector, Jr. 1984. Axial heterogeneity in the rat proximal convoluted tubule. II. Osmolality and osmotic water permeability. Am. J. Physiol. 247(Renal Fluid Electrolyte Physiol. 16):F822-F826.

34. Haberle, D. A., U. Muller, and W. Nagel. 1989. Glomerular tubular balance: Mediation by luminal hypotonicity. Miner. Electrolyte Metab. 15:108-113.

35. O'Hare, J. A., J. B. Ferriss, D. Brady, B. Twomey, and D. J. O'Sullivan. 1985. Exchangeable sodium and renin in hypertensive diabetic patients with and without nephropathy. Hypertension. 7(Suppl. II):II-43-II-48.

36. Stanton, B. A., and B. Kaissling. 1989. Regulation of renal ion transport and cell growth by sodium. Am. J. Physiol. 257(Renal Fluid Electrolyte Physiol. 26):F1-F10.

37. Heinemann, L., P. Sawicki, K. Rave, A. Hohmann, and M. Berger. 1987. Increased plasma concentrations of natriuretic peptide in type I diabetic patients with different stages of diabetic nephropathy. Proceedings of the 2 nd World Congress on Biologically Active Atrial Peptide. p. 196. (Abstr.)

38. Ortola, F. V., B. J. Ballermann, S. Anderson, R. E. Mendez, and B. M. Brenner. 1987. Elevated plasma atrial natriuretic peptide in diabetic rats. A potential mediator of hyperfiltration. J. Clin. Invest. 80:670-674. 